\title{
Wireless Positioning Using TDS-OFDM Signals in Single-Frequency Networks
}

\author{
Linglong Dai, Zhaocheng Wang, Senior Member, IEEE, Changyong Pan, Member, IEEE, and \\ Sheng Chen, Fellow, IEEE
}

\begin{abstract}
Wireless positioning using digital television (DTV) signals is a promising complementary to global positioning system due to high transmission power and wide coverage of DTV transmitters. Without changing the current infrastructure of Chinese DTV broadcasting network, this paper proposes a positioning scheme using time-domain synchronous (TDS) orthogonal frequency division multiplexing (OFDM) signals in single-frequency networks. The transmission parameter signaling (TPS) embedded in the TDS-OFDM signals is time-division multiplexed as orthogonal frequency-domain pilots, and then the time-domain pseudorandom noise sequence and the frequency-domain TPS are jointly utilized for accurate time of arrival (TOA) estimation for each transmitter. The theoretical bound of the TOA estimation accuracy is also derived. The proposed wireless positioning scheme has no impact on the normal TV program reception. Simulation results show that the positioning accuracy of less than $1 \mathrm{~m}$ can be achieved in SFN scenarios. The idea of time-frequency joint positioning can also be applied to other OFDM-based wireless systems.
\end{abstract}

Index Terms-Digital television, positioning, single-frequency networks, time-domain synchronous orthogonal frequency division multiplexing, transmission parameter signaling.

\section{INTRODUCTION}

$\mathrm{C}$ OMPARED with satellite-based navigation systems such as global positioning system (GPS), digital television (DTV) broadcasting signals have some potential advantages for low cost and yet accurate positioning. DTV signals do not suffer from the ionosphere disturbance and high Doppler effects caused by high-speed satellites, both of which degrade the performance of GPS. Operating at low frequencies, DTV

Manuscript received January 18, 2011; revised October 11, 2011; accepted December 12, 2011. Date of publication February 13, 2012; date of current version May 18, 2012. This work was supported in part by Standardization Administration of the People's Republic of China with AQSIQ Project 200910244 and in part by Tsinghua University Initiative Scientific Research Program 20091081280. This paper was presented in part at the IEEE International Conference on Communications 2011.

L. Dai, Z. Wang, and C. Pan are with the Department of Electronic Engineering and Tsinghua National Laboratory of Information Science and Technology (TNList), Tsinghua University, Beijing 100084, China (e-mail: d1107@mails.tsinghua.edu.cn; zcwang@tsinghua.edu.cn; pcy@tsinghua.edu.cn).

S. Chen is with the School of Electronics and Computer Science, Faculty of Physical and Applied Sciences, University of Southampton, Southampton SO17 1BJ, U.K., and also with the Faculty of Engineering, King Abdulaziz University, Jeddah 21589, Saudi Arabia (e-mail: sqc@ecs.soton.ac.uk).

Color versions of one or more of the figures in this paper are available online at http://ieeexplore.ieee.org.

Digital Object Identifier 10.1109/TBC.2011.2182431 signals are well-suited for urban propagation. They have a power advantage of more than $40 \mathrm{~dB}$ over GPS signals, because the received GPS signal strength is relatively weak after transmission over a great distance of more than 22,000 km [1]. A DTV broadcasting network enjoys a substantially superior geometry to what a satellite system could provide, thereby permitting position location even in the presence of blockage and in indoor environments. Due to multi-path effect on the time of arrival (TOA) estimation, it has been shown that multi-path is one of the dominant sources of the error budget for the GPS [2], while the orthogonal frequency division multiplexing (OFDM) signals used by serval digital television terrestrial broadcasting (DTTB) standards have intrinsic robustness to multi-path channels.

Rabinowitz and Spilker [3] proposed a positioning scheme using the synchronization signals specified by the American DTTB standard set forth by the advanced television systems committee (ATSC), which achieves the positioning accuracy of several meters. The positioning method based on correlation of frequency-domain pilots was presented for the European DTTB standard known as digital video broadcasting-terrestrial (DVB-T), and the achievable accuracy was in the order of meters [4]. The fundamental issues for wireless positioning using the DTV signals are the accurate TOA estimation with respect to each transmitter and transmitter identification. The transmitter identification (TxID) watermark using orthogonal Kasami sequence was proposed for the ATSC [5], [6], but such kind of watermark has not been defined by the current Chinese DTTB standard. The correlation between the received frequency-domain pilots with local pilots was proposed for the TOA estimation in DVB-T systems [7], but estimation accuracy is not satisfactory. To the best of the authors' knowledge, there exists no literature addressing the positioning issue using Chinese DTTB network whose key technology is the time-domain synchronous OFDM (TDS-OFDM) [8]. This paper intends to fill up this gap.

Regarding the positioning issue using OFDM signals, the state-of-the-art methods can be generally divided into two categories. The first one uses the traditional or improved correlationbased timing synchronization algorithms to locate the boundaries of OFDM blocks [9]-[12]. The positioning schemes used for DTV systems, including the ATSC and the DVB-T [3]-[7], all belong to this category. These schemes have low complexity but their accuracies are directly limited by the receiver sampling rate, and generally an error of several meters is expected. The methods of the other category are based on super-resolution algorithms, such as the multiple signal classification (MUSIC) [13], and the matrix pencil (MP) [14], derived from modern 
spectral estimation techniques. These methods use super resolution algorithms to extract a more accurate estimation of the first arrived path. They have better positioning accuracy but their complexity is high, which might be unacceptable for commercial receivers.

In this paper, the complete wireless positioning solution using the TDS-OFDM broadcasting signals in single-frequency networks (SFNs) is proposed for Chinese DTTB standard. The contributions of this paper are listed below:

1) The concept of regarding the transmission parameter signaling (TPS) as frequency-domain pilots is proposed, providing a new look at the TDS-OFDM signal structure;

2) Time-division multiplexing (TDM) of TPS as orthogonal frequency-domain pilots is proposed for transmitter identification and transmitter-specific TOA estimation;

3) The time-domain pseudorandom noise (PN) sequence and the frequency-domain TPS embedded in the TDS-OFDM signals are jointly utilized for positioning, which achieves better accuracy than the existing state-of-the-art positioning methods using the OFDM signals;

4) The proposed low-complexity positioning method requires no modification of the current infrastructure of the Chinese DTTB system. Thus the maximum backward system compatibility is achieved.

The remainder of this paper is organized as follows. Section II illustrates the system model for wireless positioning in the TDSOFDM-based SFNs, while Section III presents the joint timefrequency positioning algorithm using the time-domain PN sequence and the frequency-domain TPS embedded in the TDSOFDM signals. In Section IV, the performances of the proposed scheme is analysed, including the ranging accuracy, positioning time, computational complexity and system compatibility. Simulation results are presented in Section V to verify the performance of the positioning scheme. We conclude this paper in Section VI.

\section{System Model of Positioning IN THE SFN}

Most DTTB systems, including TDS-OFDM, are deployed as the SFN, where several transmitters simultaneously send identical signals in a synchronized way [15]. The SFN efficiently utilizes the spectrum with the frequency reuse factor of one. It may also decrease the outage probability and increase the coverage area, due to the inherent diversity [16]. Most OFDMbased wideband digital broadcasting systems are well-suited to the SFN operation, because of the robustness of the OFDM signal to multi-path propagation.

Without loss of generality, Fig. 1 shows the positioning topology with three transmitters in the SFN with the TDS-OFDM signals. Time difference of arrival (TDOA) of the downlink TDS-OFDM signals from multiple transmitters is used for positioning. The TDOA is the difference of two TOAs corresponding to two transmitters. As illustrated in Fig. 1, the receiver location can be uniquely calculated using two hyperbolic curves corresponding to two TDOAs.

The SFN provides some natural merits for wireless positioning, which include

1) Multiple transmitters: Multiple transmitters in the SFN can provide several TDOAs for positioning. For example, 6

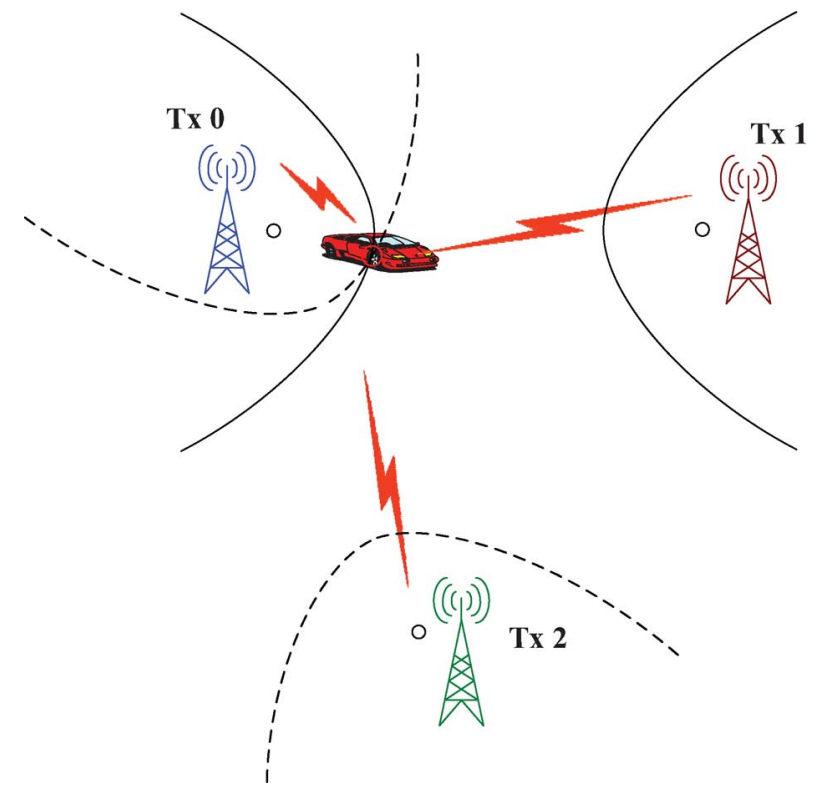

Fig. 1. Wireless positioning using TDOA in the SFN.

SFN transmitters are deployed in the TDS-OFDM broadcasting system in Hong Kong, China [17];

2) Synchronicity: The prerequisite of synchronicity among different transmitters in the SFN [15] ensures the same time basis for every transmitter, which is required by TDOA technique. Note that perfect synchronicity among different transmitters in the SFN is assumed in this paper;

3) Transmitter location: After network planning, the fixed locations of multiple transmitters in the SFN are known, and these information can be pre-stored at the receiver.

However, there are some challenges for positioning in the SFN. Two fundamental issues for positioning in the SFN have to be considered, which are

1) Accurate TOA estimation: For the TDOA-based positioning, whereby the TDOA is obtained by two estimated TOA, the fundamental issue is the accurate TOA estimation. The positioning accuracy primarily depends on the TOA estimation accuracy;

2) Transmitter identification: For positioning, it is necessary to identify different transmitters, and associate each TOA with the corresponding transmitter.

Fig. 2 compares the signal frame structure of the cyclic prefix OFDM (CP-OFDM) used in the DVB-T with that of the TDSOFDM adopted by the Chinese DTTB standard. Instead of using $\mathrm{CP}$ as the guard interval of the inverse discrete Fourier transform (IDFT) block, as in the standard CP-OFDM, the TDS-OFDM utilizes PN sequence as guard interval as well as training sequence for fast synchronization and accurate channel estimation. Therefore, frequency-domain pilots used in the CP-OFDM can be removed, leading to the increased spectral efficiency by about $10 \%-15 \%$ [17]. The cyclicity of the IDFT block however is destroyed due to the PN insertion, and this problem can be effectively solved by iterative interference cancellation [18] or cyclicity reconstruction method [19].

Both DVB-T and Chinese DTTB standards adopt the TPS embedded in the IDFT block to convey system information, 


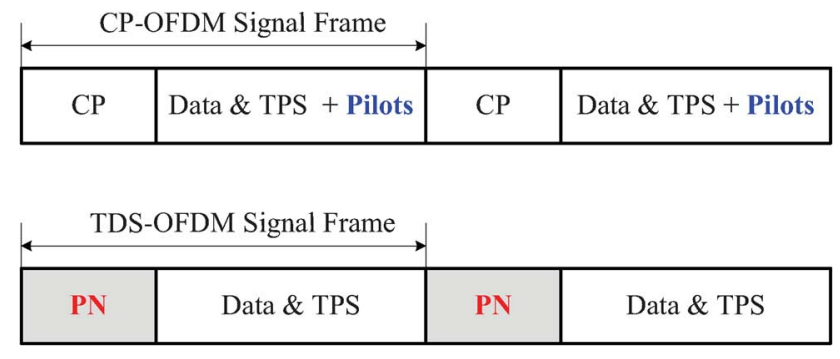

Fig. 2. Signal frame structure comparison between CP-OFDM for DVB-T and TDS-OFDM for Chinese DTTB standard.

such as constellation mapping scheme, coding rate, and interleaving mode. In this way, the TPS is used to provide necessary demodulation and decoding information at the receiver. In the TDS-OFDM, the frequency-domain IDFT block with length of 3780 consists of the normal TV program data with length of 3744 and the TPS with length of 36, i.e. the TPS occupies less than $1 \%$ of the used subcarriers. The 36 TPS symbols are equally divided into two groups located at the two sides of the IDFT block after frequency-domain interleaving. As specified by the Chinese DTTB standard [8], the TPS takes BPSK modulation (more specifically, 4QAM modulation scheme with the identical in-phase and quadrature components) for reliable reception.

The continuous-time channel impulse response (CIR) from the $m$ th transmitter in the SFN to the receiver can be modeled as a finite impulse response (FIR) filter

$$
h_{m}(t)=\sum_{l=0}^{L_{m}-1} h_{m, l} \delta_{c}\left(t-\tau_{m, l}\right),
$$

where $\delta_{c}(\cdot)$ denotes the continuous-time impulse function, $L_{m}$ is the number of paths, $h_{m, l}$ and $\tau_{m, l}$ are the attenuation coefficient and generic time delay of the $l$ th path, respectively. With the sampling period $T_{s}$ at the receiver, the discrete-time CIR can be modeled as [20]

$$
h_{m}(n)=\sum_{l=0}^{L_{m}-1} h_{m, l} \delta_{d}\left(n-n_{m, l}\right),
$$

where $\delta_{d}(\cdot)$ is the discrete-time impulse function and $n_{m, l}$ is the tapped delay corresponding to $\tau_{m, l}$.

After being passed through the multi-path wireless channel, the received time-domain signal $y(t)$ coming from $M$ visible transmitters in the SFN is given by

$$
\begin{aligned}
y(t) & =\sum_{m=0}^{M-1} x_{m}(t) \star h_{m}(t) \star \delta_{c}\left(t-\tau_{m, d}\right)+v(t) \\
& =\sum_{m=0}^{M-1} \sum_{l=0}^{L_{m}-1} h_{m, l} x_{m}\left(t-\tau_{m, l}-\tau_{m, d}\right)+v(t),
\end{aligned}
$$

where $x_{m}(t)$ is the time-domain TDS-OFDM signal sent by the $m$ th transmitter, $\tau_{m, d}$ is the signal propagation delay of the $m$ th transmitter, $\star$ denotes the linear convolution operator, and $v(t)$ is the additive white Gaussian noise (AWGN) with zero mean and the variance of $\sigma_{v}^{2}$.

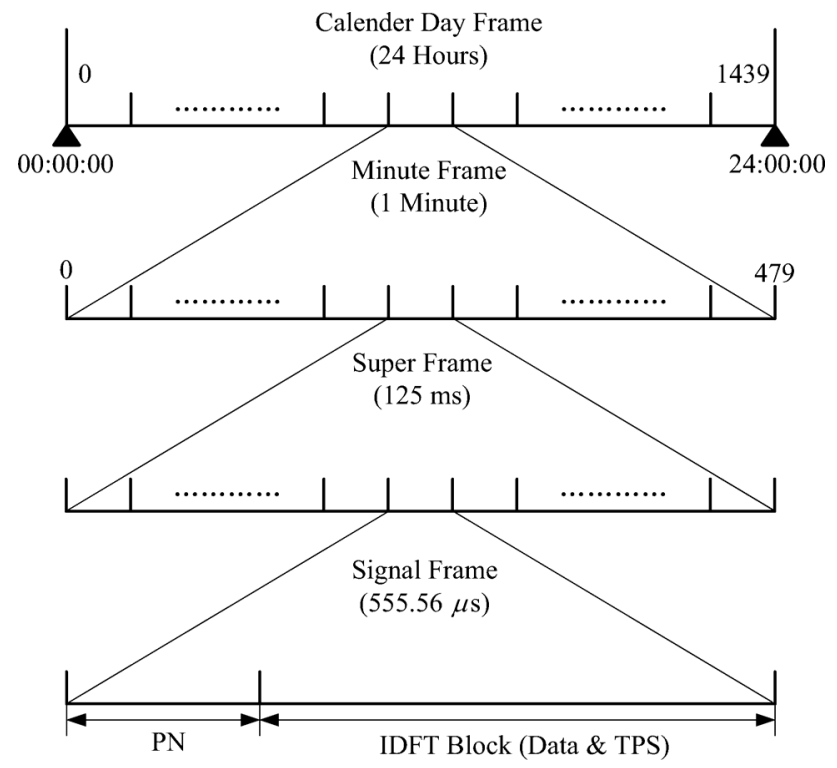

Fig. 3. Hierarchical frame structure of the TDS-OFDM system.

The transmission delay $\tau_{m, d}$ can be normalized by the sampling period $T_{s}$ as

$$
\theta_{m}=\frac{\tau_{m, d}}{T_{s}}=\theta_{m, I}+\theta_{m, F},
$$

where $\theta_{m, I}$ is the normalized integer delay, and $\theta_{m, F} \in$ $(-0.5,+0.5]$ is the normalized fractional delay. The TDOA between the $m$ th transmitter and the $n$th transmitter is $\left(\tau_{m, d}-\tau_{n, d}\right)$. Thus the corresponding distance difference for positioning is

$$
D_{m, m}=\left(\tau_{m, d}-\tau_{n, d}\right) c=\left(\theta_{m, I}+\theta_{m, F}-\theta_{n, I}-\theta_{n, F}\right) T_{s} c,
$$

where $c=3 \times 10^{8} \mathrm{~m} / \mathrm{s}$ is the speed of light in the free space.

\section{Proposed Positioning Using TDS-OFDM Signals}

In this section, the TOA estimation in the TDS-OFDM system is firstly investigated. Then, the TDM of the TPS as orthogonal frequency-domain pilots for the TDS-OFDM signal is proposed, based on which the time-frequency joint positioning scheme is detailed.

\section{A. Overview of TOA Estimation in the TDS-OFDM System}

One of the key features of the TDS-OFDM system is the hierarchical frame structure, as shown in Fig. 3. From the top to the bottom, there are Calendar Day Frame (starting from 00:00:00 every day, and ending at 24:00:00, Pacific Standard Time or Beijing Time), Minute Frame lasting exactly one minute, Super Frame with the fixed duration of $125 \mathrm{~ms}$, and Signal Frame occupying $555.56 \mu \mathrm{s}$. One promising application of the hierarchical frame structure is the power saving of the TDS-OFDM receiver at the physical layer [21].

When considering positioning with the TDS-OFDM signals, the hierarchical frame structure is especially useful for accurate TOA estimation due to the exact alignment of the signal frame with the absolute time (Pacific Standard Time or Beijing Time). The idea is that, for the synchronous SFN, the receiver can easily 


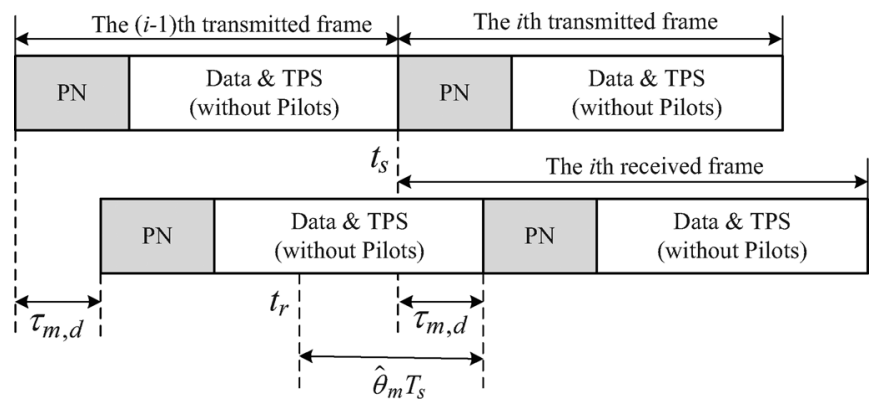

Fig. 4. Transmitted signal and received signal with the propagation delay of $\tau_{m, d}$.

know the current frame number. Thus, the exact transmitting time of this signal frame is known.

As illustrated in Fig. 4, starting from any time stamp $t_{r}$ at the receiver, the absolute time of the beginning point of the $i$ th TDS-OFDM signal frame can be calculated by the frame synchronization algorithm after $\widehat{\theta}_{m}$ samples. The frame number is known after frame synchronization. Consequently, the absolute transmitting time stamp $t_{s}$ is obtained. Finally, the TOA can be calculated as

$$
\tau_{m, d}=t_{r}+\widehat{\theta}_{m} T_{s}-t_{s}
$$

In a real-world SFN, the TDS-OFDM receiver is unlikely to be perfectly synchronized with the transmitters, i.e. the time stamp $t_{r}$ is not ideally aligned with the absolute time. This will lead to a constant error for the TOA estimation. However, this problem can be resolved by using TDOA for positioning, since the constant error embedded in two TOA estimates is removed by subtraction.

\section{B. TDM of TPS as Orthogonal Frequency-Domain Pilots}

The TDS-OFDM signal is a skillful combination of time-frequency processing, i.e. the time-domain processing of the PN sequence and the frequency-domain processing of the IDFT block are carefully considered together. Conventionally, the TPS embedded in the IDFT block of the TDS-OFDM signal is only used to provide demodulation and decoding information at the receiver. The TDS-OFDM is regarded as a scheme without frequency-domain pilots, and the PN sequence provides the only training information. However, as shown in Fig. 5, the TPS may be considered as frequency-domain pilots based on the following observations:

1) Robust transmission: Since the TPS is BSPK modulated, its demodulation threshold is much lower than high-order modulation schemes, such as 4QAM/16QAM/64QAM. Therefore, when the DTV program data with high-order constellation mapping scheme can be correctly received, the TPS can be demodulated almost perfectly;

2) Invariance: As the system information, the TPS does not change frequently. Typically, the TDS-OFDM broadcasting system uses the same TPS for several hours, or even dozens of days. Once the TPS has been correctly detected in the previous signal frame, it can be regarded as known symbols in the following signal frames, whereby

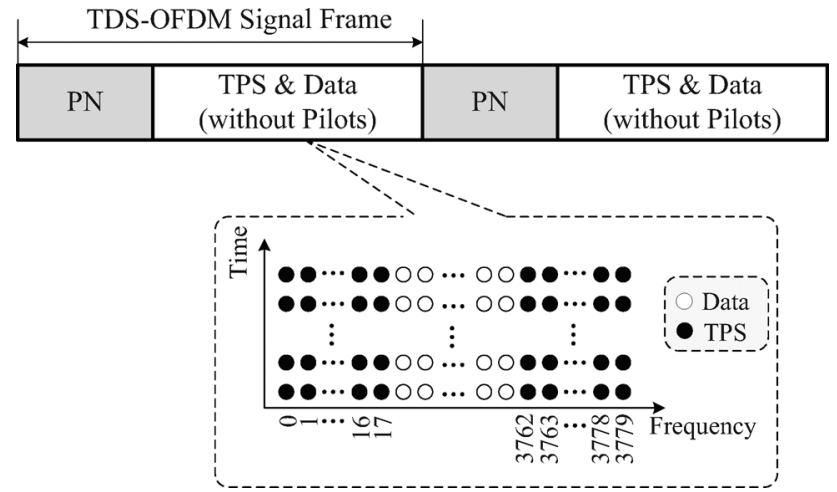

Fig. 5. New look at the TDS-OFDM signal: regarding TPS as frequency-domain pilots.

the TPS detection is unnecessary any more. This is the main reason why the TPS can be treated as frequency-domain pilots;

3) Unchanged location: Unlike the scattered pilots whose location varies frame-by-frame in DVB-T [23], the TPS in TDS-OFDM occupies the unchanged location in the frequency domain.

For the purpose of transmitter identification and the transmitter-specific TOA estimation, the TDM of TPS is proposed in the SFN using the TDS-OFDM signals, which can be defined as

$$
P_{m, k}^{(i)}= \begin{cases} \pm 1, & (i-m) \bmod (M-1)=0, k \in \Psi \\ 0, & \text { others }\end{cases}
$$

where mod denotes the modulo operator, $P_{m, k}^{(i)}$ denotes the TPS over the $k$ th subcarrier for the $m$ th transmitter in the $i$ th signal frame, and

$$
\Psi=\{0,1, \ldots, Q\} \cup\{\Delta, \Delta+1, \ldots, \Delta+Q\}
$$

is the index set of the TPS, while $Q=17$ and $\Delta=3762$ according to [8]. In the TDS-OFDM systems, after the initialization step, the receiver knows the exact frame number $i$ of the received signal frame due to the hierarchical frame structure, as shown in Fig. 3. Thus, the frame number $i$ can be easily associated with the $m$ th transmitter due to the TDM of TPS as define by (7).

The time-division multiplexed TPS is illustrated in Fig. 6, using the case of four transmitters $(M=4)$ as an example. In each signal frame, only one transmitter sends the TPS, while other transmitters convey zeros at the subcarriers originally occupied by the TPS. As depicted in Fig. 6 and mathematically defined in (7), the TDM of TPS provides orthogonal frequencydomain pilots in the TDS-OFDM signals, offering the orthogonality for transmitter identification and the TOA estimation using the TPS.

\section{Time-Frequency TOA Estimation Algorithm}

We now propose the time-frequency TOA estimation algorithm using the time-domain PN sequence and the frequency-domain TPS (pilots) for the TDS-OFDM-based SFNs. 


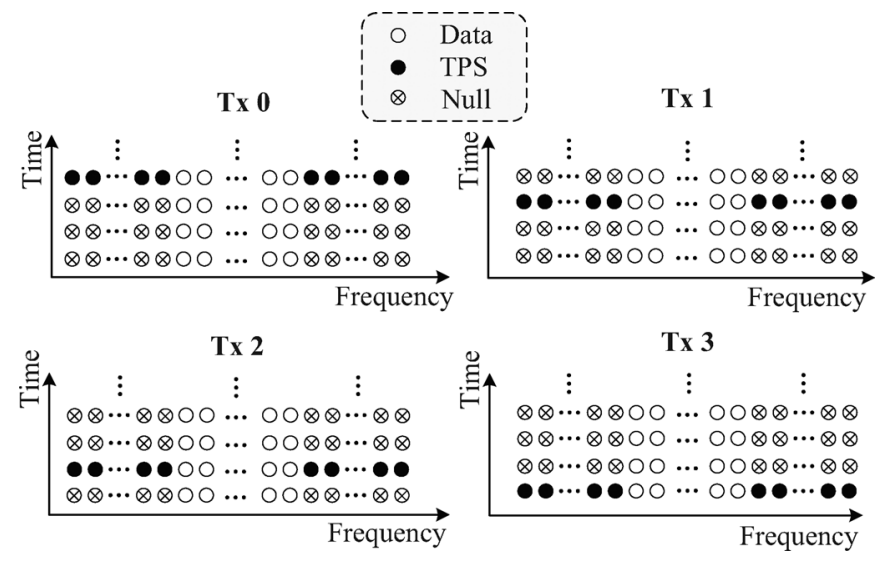

Fig. 6. Time-division multiplexing (TDM) of TPS for transmitter identification and TOA estimation in the SFN.

The transmitted continuous-time time-domain IDFT block can be expressed as

$$
x_{m}(t)=\frac{1}{\sqrt{N}} \sum_{k=0}^{N-1} X_{m, k} e^{j 2 \pi f_{k} t},
$$

where $N$ is the IDFT block length, $X_{m, k}$ is the frequency-domain symbol over the $k$ th subcarrier for the $m$ th transmitter, $f_{k}=k f_{0}$ is the frequency of the $k$ th subcarrier, while $f_{0}=$ $1 / N T_{s}$ is the subcarrier frequency spacing. Note that $N T_{s}$ is the IDFT block duration. The corresponding received signal in (3) can be rewritten as

$$
\begin{aligned}
y(t)=\frac{1}{\sqrt{N}} \sum_{m=0}^{M-1} \sum_{l=0}^{L_{m}-1} \sum_{k=0}^{N-1} h_{m, l} X_{m, k} \\
\times e^{j 2 \pi \frac{k}{N T_{s}}\left(t-\tau_{m, l}-\tau_{m, d}\right)}+v(t) .
\end{aligned}
$$

Sampling the received signal with the time interval $T_{s}$ generates the discrete-time samples

$$
\begin{array}{r}
y(n)=\frac{1}{\sqrt{N}} \sum_{m=0}^{M-1} \sum_{l=0}^{L_{m}-1} \sum_{k=0}^{N-1} h_{m, l} X_{m, k} e^{j \frac{2 \pi}{N} k\left(n-n_{m, l}\right)} \\
\times e^{-j \frac{2 \pi}{N} k \theta_{m, I}} e^{-j \frac{2 \pi}{N} k \theta_{m, F}}+v(n),
\end{array}
$$

where $v(n)$ is the noise term. The corresponding frequency-domain signal over the $k$ th subcarrier is obtained after the discrete Fourier transform (DFT)

$$
Y_{k}=\sum_{m=0}^{M-1}\left(H_{m, k} X_{m, k} e^{-j \frac{2 \pi}{N} k \theta_{m, F}}\right) e^{-j \frac{2 \pi}{N} k \theta_{m, I}}+V_{k},
$$

where $V_{k}$ is the DFT of $v(n)$, and $H_{m, k}$ is the channel frequency response (CFR) defined as

$$
H_{m, k}=\sum_{l=0}^{L_{m}-1} h_{m, l} e^{-j \frac{2 \pi}{N} k n_{m, l}} .
$$

It is clear that the impacts of the transmission delay $\theta_{m}$ on the received signal are twofold: The time shift by $\theta_{m, I}$ samples in the time domain and the linear phase shift by $-(2 \pi / N) k \theta_{m, F}$ in the frequency domain.

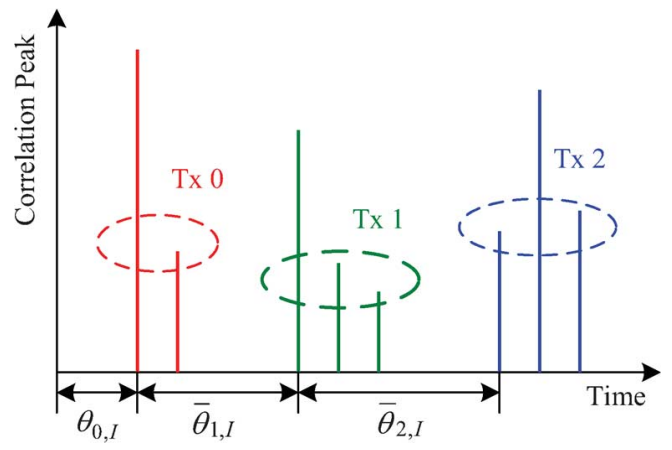

Fig. 7. Correlation peaks for integer TOA estimation.

Integer TOA Estimation Using Time-Domain PN Sequence: After the interference between the PN sequence and the IDFT data block has been removed by the standard TDS-OFDM receiver [18], the received PN sequence is denoted by $r(n)$. Then the integer delays corresponding to different transmitters can be roughly estimated by the time-domain sliding correlation between the $r(n)$ and the local PN sequence $p(n)$

$$
c(d)=\sum_{n=0}^{N_{p}-1} r^{*}(n-d) p(n),
$$

where $N_{p}$ is the length of the PN sequence, $r^{*}(n)$ denotes the conjugate of $r(n)$, and $c(d)$ is the correlation result corresponding to the slide of $d$. Due to the near-ideal autocorrelation property of the PN sequence, the correlation peak is confirmed when the local PN sequence is aligned with the received PN sequence. Thus, as illustrated in Fig. 7, correlation peaks appear at the slide values

$$
d_{m, l}=\theta_{m, I}+n_{m, l}, \quad 0 \leq m \leq M-1, \quad 0 \leq l \leq L_{m}-1 .
$$

According to [22], the correlation-based method (14) yields the timing accuracy within the range $\left(-T_{\text {sample }} / 2,+T_{\text {sample }} / 2\right]$, where $T_{\text {sample }}$ is the actual sampling period used. In the TDSOFDM receivers, the received signal is usually over-sampled by a factor of four, i.e. $T_{\text {sample }}=T_{s} / 4$, which results in the timing accuracy in the range of $\left(-T_{s} / 8,+T_{s} / 8\right]$. Without loss of generality, $d_{0}=\theta_{0, I}$ is assumed to be the location of the first significant correlation peak. After compensating for the first large integer delay $\theta_{0, I}$, the received DFT signal block becomes

$$
Y_{k}=\sum_{m=0}^{M-1}\left(H_{m, k} X_{m, k} e^{-j \frac{2 \pi}{N} k \theta_{m, F}}\right) e^{-j \frac{2 \pi}{N} k \bar{\theta}_{m, I}}+V_{k},
$$

where $0 \leq k \leq N-1$, and $\bar{\theta}_{m, I}=\theta_{m, I}-\theta_{0, I}$ is the differential integer delay of the $m$ th transmitter with respect to the first integer delay $\theta_{0, I}$.

However, obtaining correlation peaks is not sufficient for integer delay estimation. The reason lies in two aspects. Firstly, as indicated by (15), the number of correlation peaks in (14) is usually larger than the number of visible transmitters in multi-path scenarios. Thus, there is no information about which peak is associated with a specific transmitter. Secondly, there is no way to distinguish the coincided correlation peaks corresponding to different transmitters. For example, if the receiver is situated 
at the middle point between two transmitters, the two identical TOAs may be observed as a single correlation peak. These problems can be solved by using the proposed time-division multiplexed TPS as follows.

Owing to the proposed TDM of TPS, the received TPS $Y_{k}^{(i)}$ in the $i$ th signal frame can be rewritten as

$$
\begin{aligned}
Y_{k}^{(i)} & =\sum_{m=0}^{M-1}\left(H_{m, k}^{(i)} P_{m, k}^{(i)} e^{-j \frac{2 \pi}{N} k \theta_{m, F}}\right) e^{-j \frac{2 \pi}{N} k \bar{\theta}_{m, I}}+V_{k} \\
& =\left\{\begin{array}{cc}
H_{m, k}^{(i)} P_{m, k}^{(i)} e^{-j \frac{2 \pi}{N} k\left(\bar{\theta}_{m, I}+\theta_{m, F}\right)} & i=u M+m, k \in \Psi, \\
+V_{k}, & \text { others, } \\
V_{k}, &
\end{array}\right.
\end{aligned}
$$

where $H_{m, k}^{(i)}$ denotes the CFR in the $i$ th frame, and $u$ is an arbitrary positive integer. The result of (17) indicates that the TPS of the $m$ th transmitter is received at the $(u M+m)$ th signal frame.

Since the TPS can be regarded as the known frequency-domain pilots, $P_{m, k}^{(i)}$ in (17) is known for $k \in \Psi$. According to [24], channel state information can be assumed to be known when the impact of transmission delay on the received signal is investigated. Thus the received TPS associated with the $m$ th transmitter can be simplified below by removing the frame index $i$

$$
R_{k}=e^{-j \frac{2 \pi}{N} k\left(\bar{\theta}_{m, I}+\theta_{m, F}\right)}+W_{k}, \quad k \in \Psi,
$$

where $W_{k}=V_{k} /\left(H_{m, k} P_{m, k}\right)$ is the complex-valued Gaussian white noise term with

$$
E\left\{W_{k}^{*} W_{k}\right\}=\frac{\sigma_{v}^{2}}{E\left\{\left(H_{m, k} P_{m, k}\right)^{*}\left(H_{m, k} P_{m, k}\right)\right\}}=\sigma^{2}=\frac{1}{\gamma},
$$

with $E\{\cdot\}$ being the expectation operator, and $\gamma$ being the average signal-to-noise ratio (SNR).

To distinguish the correlation peaks obtained using (14), the TPS can be used to roughly estimate the differential integer delay $\bar{\theta}_{m, I}$ of the $m$ th transmitter according to

$$
\widehat{\bar{\theta}}_{m, I}=\frac{N}{2 \pi} \arg \left\{\sum_{k=0}^{Q-2} R_{k}^{*} R_{k+1}+\sum_{k=\Delta}^{\Delta+Q-2} R_{k}^{*} R_{k+1}\right\},
$$

where $0 \leq m \leq M-1$, and $\arg \{x\}$ denotes the angle of the complex-valued signal $x$.

The $M$ differential integer delays can be obtained after the processing in (20) over $M$ adjacent TDS-OFDM signal frames, whereby the TPS from only one transmitter is received in each frame. These $M$ values can be used to distinguish the corresponding $M$ correlation peaks obtained in (14) by associating the results in (14) with the nearest counterparts in (20). Thus the final integer TOA estimation is given by

$$
\widehat{\theta}_{m, I}=\min _{d_{m, l}}\left|d_{m, l}-d_{0}-\widehat{\bar{\theta}}_{m, I}\right|, \quad 1 \leq m \leq M-1 .
$$

The above mechanism can solve the problem caused by the number of correlation peaks being larger than $M$. Moreover, it also works under the situation where different transmitters share the same correlation peak, since these transmitters can be identified by the results from (20). Notice that the integer delay estimation accuracy within the range $\left(-T_{s} / 8,+T_{s} / 8\right]$ is guaranteed by the near-ideal autocorrelation of the time-domain PN sequence, not by the results of (20).

Up to now, the integer delay estimation has been obtained by combining the results from (14) and (20). For the actual communication purpose, it is sufficient to locate the beginning of each transmission block within one sampling period for correct demodulation, since the fractional delay is inherently compensated for by the one-tap frequency-domain equalization [25]. In other words, the fractional delay $\theta_{m, F}$ is usually ignored for data recovery. However, for the positioning purpose, the fractional delay must be estimated accurately in order to achieve high positioning accuracy. This is because the estimation accuracy provided by the integer delay estimation alone is strictly limited by the sampling period. For the TDS-OFDM system with the sampling rate of $7.56 \mathrm{MHz}$, the ranging error turns out to be $\left(T_{s} / 2\right) c$, which is about $20 \mathrm{~m}$. Even for the over-sampling by the factor of four, namely with the sampling rate of $30.24 \mathrm{MHz}$, the estimation error is about $5 \mathrm{~m}$, which is not sufficient for accurate positioning.

Fractional TOA Estimation Using Frequency-Domain TPS: After compensating for the integer delay $\theta_{m, I}$, the received TPS associated with the $m$ th transmitter can be expressed as

$$
R_{k}=e^{-j \frac{2 \pi}{N} k \theta_{m, H}}+W_{k}, \quad k \in \Psi .
$$

The fractional delay $\theta_{m, F}$ can be estimated according to

$$
\widehat{\theta}_{m, F}=\frac{N}{2 \pi \Delta} \arg \left\{\sum_{k=0}^{N-1-\Delta} R_{k}^{*} R_{k+\Delta}\right\} .
$$

The estimation range of $\widehat{\theta}_{m, F}$ in $(23)$ is $(-(N / 2 \Delta),+(N / 2 \Delta)]$, which is larger than the range of the actual fractional delay $\theta_{m, F} \in(-1 / 8,+1 / 8]$. The estimation accuracy will be derived in the next section.

After the integer and fractional delays have been acquired in (21) and (23), respectively, the TOA estimate $\widehat{\tau}_{m, d}$ for the $m$ th transmitter can be obtained according to

$$
\widehat{\tau}_{m, d}=\left(\widehat{\theta}_{m, I}+\widehat{\theta}_{m, F}\right) T_{s}, \quad 0 \leq m \leq M-1 .
$$

Due to the TDM of TPS, the receiver knows that the TPS in the current received signal frame is transmitted by which transmitter. Thus the TOA estimation obtained in (24) can easily be associated with the correct $m$ th transmitter. Then, the fixed location of the $m$ th transmitter pre-stored at the receiver and the corresponding TOA $\widehat{\tau}_{m, d}$ can be used for positioning.

As mentioned in Section III-A, the TDOA is used to remove the common TOA estimation error in $\left\{\widehat{\tau}_{m, d}\right\}_{m=0}^{M-1}$ caused by the non-ideal synchronization between the receiver and transmitters. Let $\widehat{D}_{m}$ and $\widehat{D}_{n}$ be the TOA-based ranging results for the $m$ th and $n$th transmitters, respectively. Then, the distance difference $\widehat{D}_{m, n}$ associated with the $m$ th and $n$th transmitters is given by

$$
\widehat{D}_{m, n}=\widehat{D}_{m}-\widehat{D}_{n}=\left(\widehat{\tau}_{m, d}-\widehat{\tau}_{n, d}\right) c, \quad 0 \leq m, n \leq M-1 .
$$

After at least three TOA measurements and the corresponding two distance differences in (25) have been obtained, the location of the receiver can be uniquely determined using the available localization algorithms summarized in [26]. For example, the 
closed-form solutions proposed in [27], [28] or the iterative localization method using Taylor-series given in [29] can be used to finally determine the receiver location.

\section{Performance AnAlysis}

We now analyse the performance of the proposed time-frequency positioning algorithm, including the ranging accuracy, positioning time, computational complexity, and system compatibility.

\section{A. TOA-Based Ranging Accuracy}

Since the integer TOA can be estimated with extremely high accuracy due to the good autocorrelation property of the PN sequence [22], the positioning accuracy of the proposed time-frequency positioning method mainly depends on the accuracy of the fractional TOA estimation (23). By denoting $\Omega=(2 \pi / N) \theta_{m, F},(23)$ can be rewritten as

$$
\widehat{\Omega}=\frac{1}{\Delta} \arg \{\widehat{D}(\Delta)\},
$$

where

$$
\widehat{D}(\Delta)=\sum_{k=0}^{N-1-\Delta} R_{k}^{*} R_{k+\Delta} .
$$

According to [30], the estimator (26) is completely characterized by its state space parameter obtained for processing the noise-free signal, and this state space parameter is given by

$$
\Omega=\frac{1}{\Delta} \arg \{D(\Delta)\}=\frac{1}{\Delta} \Im\{\ln \{D(\Delta)\}\},
$$

where $\Im\{x\}$ denotes imaginary part of $x$, and

$$
D(\Delta)=\sum_{k=0}^{N-1-\Delta}\left(e^{j k \Omega}\right)^{*} e^{j(k+\Delta) \Omega}=(N-\Delta) e^{j \Delta \Omega} .
$$

Since $\Im\{\cdot\}$ is a linear operation and the derivative of $\ln \{x\}$ is $1 / x$, we have

$$
\delta \Omega=\frac{1}{\Delta} \Im\left\{\frac{\delta D(\Delta)}{D(\Delta)}\right\},
$$

and the unexpected error due to the noise in (27) is presented by

$\delta D(\Delta)=\sum_{k=0}^{N-1-\Delta}\left(e^{-j k \Omega} W_{k+\Delta}+e^{j(k+\Delta) \Omega} W_{k}^{*}+W_{k}^{*} W_{k+\Delta}\right)$.

Substituting (31) into (30) yields

$$
\delta \Omega=\frac{1}{\Delta(N-\Delta)} \sum_{k=0}^{N-1-\Delta} \nu_{k}
$$

where

$\nu_{k}=\Im\left\{e^{-j(k+\Delta) \Omega} W_{k+\Delta}+e^{j k \Omega} W_{k}^{*}+e^{-j \Delta \Omega} W_{k}^{*} W_{k+\Delta}\right\}$.

Since $W_{k}$ and $W_{k+\Delta}$ are uncorrelated, and $E\left\{W_{k}\right\}=$ $E\left\{W_{k+\Delta}\right\}=0$, the expectation of $\delta \Omega$ should be

$$
E\{\delta \Omega\}=\frac{1}{\Delta(N-\Delta)} \sum_{k=0}^{N-1-\Delta} E\left\{\nu_{k}\right\}=0 .
$$

Therefore, the error variance can be derived as (35) on the bottom of this page.

$$
\begin{aligned}
\operatorname{var}\{\delta \Omega\}=E\{( & \left.\frac{1}{\Delta(N-\Delta)} \sum_{k=0}^{N-1-\Delta} \nu_{k}\right) \\
& \left.\times\left(\frac{1}{\Delta(N-\Delta)} \sum_{l=0}^{N-1-\Delta} \nu_{l}\right)\right\} \\
= & \frac{1}{\Delta^{2}(N-\Delta)^{2}} \sum_{k=0}^{N-1-\Delta} \sum_{l=0}^{N-1-\Delta} E\left\{\nu_{k} \nu_{l}\right\} .
\end{aligned}
$$

As proved by (43) in Appendix, $E\left\{\nu_{k} \nu_{l}\right\}$ in (35) is $E\left\{\nu_{k} \nu_{l}\right\}=$ $\sigma^{2} \delta_{k, l} \cos ((k-l) \Omega)+\left(\sigma^{4} / 2\right) \delta_{k, l}$, where $\delta_{k, l}=1$ for $k=l$ and $\delta_{k, l}=0$ for $k \neq l$. By substituting $E\left\{\nu_{k} \nu_{l}\right\}$ into (35), we can finally derive the error variance as (36) on the bottom of this page.

$$
\begin{aligned}
\operatorname{var}\{\delta \Omega\}= & \frac{1}{\Delta^{2}(N-\Delta)^{2}} \sum_{k=0}^{N-1-\Delta} \sum_{l=0}^{N-1-\Delta} \\
& \times\left(\sigma^{2} \delta_{k, l} \cos ((k-l) \Omega)+\frac{\sigma^{4}}{2} \delta_{k, l}\right) \\
= & \frac{1}{\Delta^{2}(N-\Delta)^{2}}\left(\sigma^{2}(N-\Delta)+\frac{\sigma^{4}}{2}(N-\Delta)\right) \\
= & \frac{1}{\Delta^{2}(N-\Delta)}\left(\frac{1}{\gamma}+\frac{1}{2 \gamma^{2}}\right) .
\end{aligned}
$$

Using the result of (25), the TOA-based ranging accuracy of the proposed scheme, in terms of root mean square error (RMSE), is therefore given by

$$
\operatorname{RMSE}\left(\widehat{D}_{m}\right)=\frac{N T_{s} c}{2 \pi \Delta} \sqrt{\frac{1}{N-\Delta}\left(\frac{1}{\gamma}+\frac{1}{2 \gamma^{2}}\right)} .
$$

It should be pointed out that the positioning accuracy will depend on the above ranging accuracy as well as the localization algorithm employed.

\section{B. Positioning Time}

As is evident from the preceding discussion and analysis, the TDM of TPS is implemented per every TDS-OFDM signal frame, and the time-frequency TOA estimation requires $M$ TDS-OFDM signal frames to acquire the $M$ TOAs in (24) for the final positioning. That is, the location estimation can be updated every $M$ TDS-OFDM signal frames. Therefore, if the receiver has been synchronized with the transmitter, the positioning time $T_{P}$ of the proposed method is given by

$$
T_{P}=M T_{F},
$$

where $T_{F}$ denotes the duration of one TDS-OFDM signal frame. On the other hand, if the receiver has not been synchronized with the transmitter, the average time for the first positioning is given by

$$
\begin{aligned}
T_{P} & =\int_{0}^{T_{F}}\left(T_{P N}+T_{F}-t\right) \frac{1}{T_{F}} d t+\left(M T_{F}-T_{P N}\right) \\
& =\left(M+\frac{1}{2}\right) T_{F},
\end{aligned}
$$


TABLE I

COMPUTATIONAL COMPLEXITY COMPARISON

\begin{tabular}{cc}
\hline \hline Method & Complexity \\
\hline Correlation-Based Synchronization [9]-[12] & $O\left(N_{p}\right)$ \\
Super-resolution Algorithm [13], [14] & $O\left(N_{p}^{3}\right)$ \\
Proposed Scheme & $O\left(N_{p}+Q\right)$ \\
\hline
\end{tabular}

where $T_{P N}$ is the PN sequence duration. Averagely, $T_{F} / 2$ more time is required for the additional PN tracking process when the synchronization is unavailable for the first positioning.

For the TDS-OFDM systems, $T_{F}$ is $589.3 \mu \mathrm{s}$ in the PN420 mode, and $692.6 \mu$ s in the PN945 mode [8]. Therefore, the first positioning time of the proposed positioning algorithm is much shorter than 10 to 15 minutes achievable in GPS [1]. Thus fast positioning using the TDS-OFDM signals can be realized.

\section{Computational Complexity}

The complexity of the conventional positioning methods based on the detection of correlation peak [9]-[12] have a low complexity of $O\left(N_{p}\right)$, where $N_{p}$ is the total number of used data symbols for positioning. However, these methods can only achieve an accuracy of several meters, which is not sufficient for positioning.

The positioning methods based on the super-resolution algorithms [13], [14] achieve an attractive estimation accuracy of several decimeters at the cost of high complexity. Specifically, the MUSIC-based algorithm [13] needs $N_{p}^{3}+N_{p}^{2}$ multiplications for each iteration step, while the MP-based method [14] requires $9 N_{p}^{3}$ multiplications. Thus, the complexity of this class of methods is $O\left(N_{p}^{3}\right)$.

The absolute integer TOA estimation based on the sliding correlation (14) needs $N_{p}$ multiplications, and the differential integer TOA estimation in (20) requires $2 Q$ multiplications, while the fractional TOA estimation in (23) demands $Q+1$ multiplications. Therefore, the complexity of the proposed method is $O\left(N_{p}+Q\right)$, which is much lower than that of the super-resolution-based methods and is similar to that of the conventional positioning methods. We will demonstrate latter that the proposed scheme is capable of achieving a high positioning accuracy.

Table I summarizes the computational complexity of these three positioning schemes.

\section{System Compatibility}

At the transmitter part, the TDM of TPS requires that only one transmitter sends the TPS in a specific signal frame, while the TPS is transmitted by every transmitter in each signal frame in the conventional SFNs. This trivial modification can be easily implemented by an on-off switch, without changing the current infrastructure of the TDS-OFDM systems. At the receiver part, the TDM of TPS has no impact on the TPS detection and normal TV program reception at the receiver, since the TDM of TPS still guarantees the TPS reception in every signal frame. Therefore, maximum backward system compatibility is achieved.

\section{SimUlation RESUltS AND Discussions}

Computer simulations were carried out to evaluate the performance of the proposed positioning scheme. The main system parameters were configured to be consistent with the

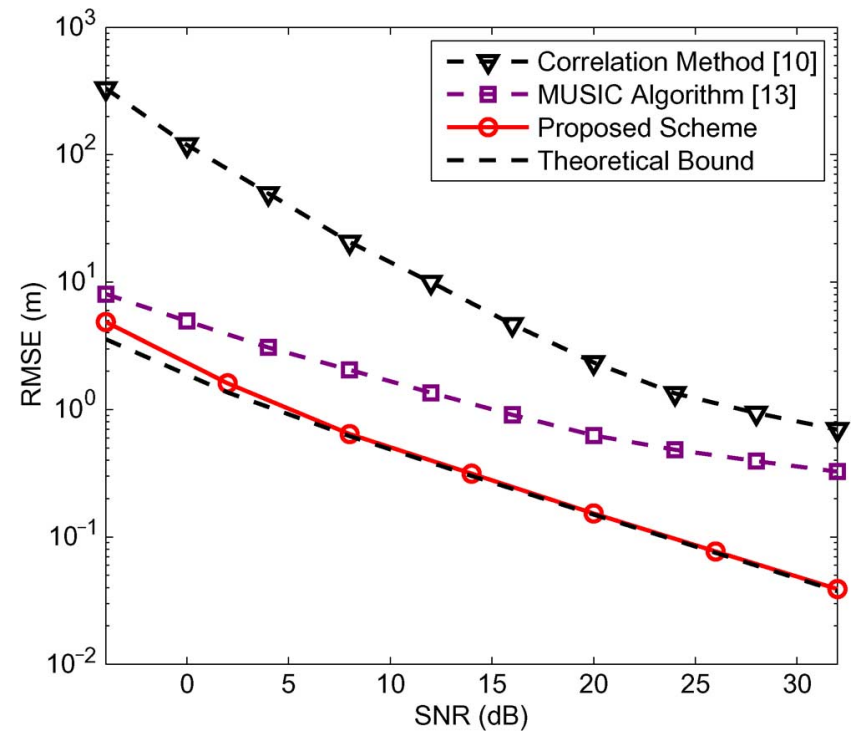

Fig. 8. Ranging accuracy comparison of three schemes over AWGN channel.

TDS-OFDM system specified in [8]. The received signal was over-sampled by the factor of four. Four transmitters were assumed to be visible in the simulated SFN, i.e. $M=4$. After obtaining three TDOAs, the closed-form localization algorithm proposed in [28] was used to determine the receiver location. The AWGN channel, the Indoor A multi-path channel defined by ITU [31] and the 8th State Administration of Radio Film and Television channel (SARFT 8) for Chinese DTTB test [18] were used in the simulation. The maximum delay spread of the Indoor A channel is only $0.31 \mu \mathrm{s}$, while the SARFT 8 channel has an $0 \mathrm{~dB}$ echo with the delay of $31.8 \mu$ s which represents a severely frequency-selective fading channel. The SNR was defined as the ratio of the transmitted signal power to the channel AWGN power.

Fig. 8 presents the TOA-based ranging accuracy comparison for three schemes, in terms of RMSE over the AWGN channel, where the theoretical bound (37) is also included as the benchmark for comparison. When the SNR was $20 \mathrm{~dB}$, the ranging error was about $2.4 \mathrm{~m}$ for Mensing's correlation-based method [10], $0.62 \mathrm{~m}$ for the MUSIC-based super-resolution algorithm [13], and $0.15 \mathrm{~m}$ for the proposed scheme. It can be seen that the proposed scheme achieved the best performance. Moreover, its ranging error approached the theoretical bound when the SNR was above $8 \mathrm{~dB}$, while only deviated slightly from the theoretical bound for lower SNR values. The reason for the improved performance is due to the fact that the proposed time-frequency TOA estimation algorithm utilizes TPS embedded in TDS-OFDM signals as frequency-domain pilots and, therefore, is able to use both the time- and frequency-domain resources. By contrast, the conventional schemes can only rely on either the time- or frequency-domain resources.

Fig. 9 shows the TDOA-based positioning accuracy achieved by the proposed scheme with the localization algorithm [28] over the two multi-path channels. For reference, both the simulated positioning accuracy and the theoretical bound over the AWGN channel are included in Fig. 9, where the ranging error of $0.15 \mathrm{~m}$ generated the positioning error of $0.082 \mathrm{~m}$ over 


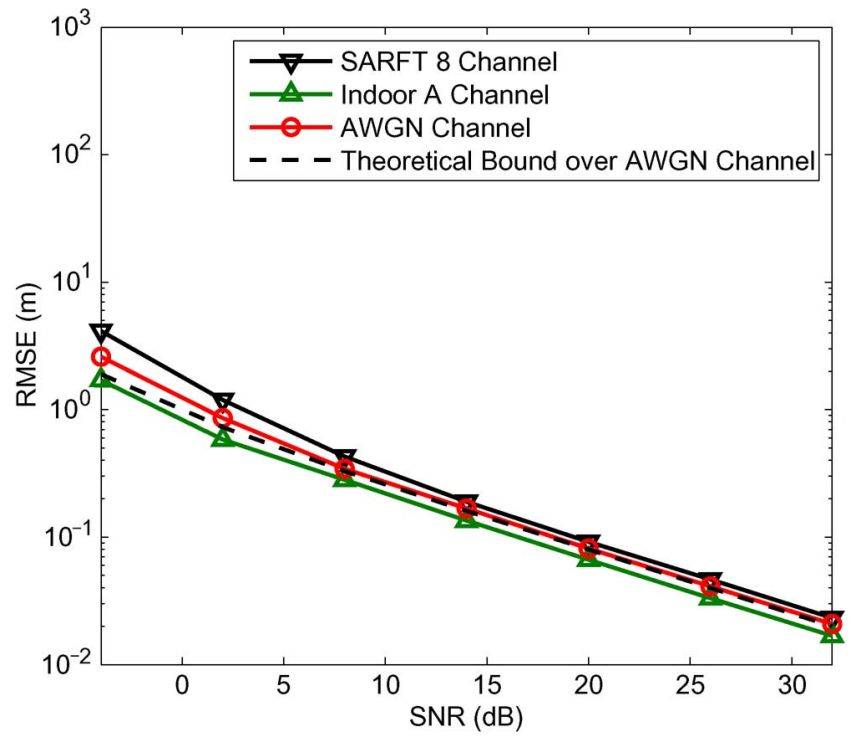

Fig. 9. Positioning accuracy of the proposed scheme over multi-path as well as AWGN channels.

the AWGN channel with the SNR value of $20 \mathrm{~dB}$. Under the same SNR value, the positioning accuracy was $0.092 \mathrm{~m}$ over the SARFT 8 channel, and $0.067 \mathrm{~m}$ over the Indoor A channel. It is interesting to see that, compared with the AWGN channel, a better positioning performance was obtained for the Indoor $\mathrm{A}$ multi-path channel. The reason may be explained as follows. The TPS are located at the two sides of the used subcarriers (see Fig. 5), and the CFR over these subcarriers corresponds to the low-frequency response of the channel. The Indoor A channel [31] fortunately has a good low-frequency response, namely $\left|H_{m, k}\right| \geq 1$ for $k \in \Psi$. This results in the higher equivalent SNR over the subcarrier set $k \in \Psi$ than the averaged channel SNR, thus yielding a better positioning performance.

The above simulation experiments demonstrate that the proposed positioning scheme outperforms some existing methods. The experimental conditions in the above simulations, however, imply that all the transmitters have the same SNR value, which is generally not the case in real-world SFNs. We then simulated a more realistic TDS-OFDM system based on the actually SFN deployed in Hong Kong [32], which consists of six transmitters. The simulation was set up according to [32], whereby two principal stations with the maximum power of $1000 \mathrm{~W}$ and other four principal stations with the maximum transmitting power of $320 \mathrm{~W}$ were launched. The farthest station away from the user was $40 \mathrm{~km}$, the pathloss effect was considered using the pathloss exponent of 3.8 in urban areas, and the hearability issue was simulated by assuming that two out of the six stations were invisible to the user due to severe blockage and consequently very weak signals coming from these two particular stations. For simplicity, free-space propagation was considered in the simulation. Fig. 10 shows the positioning accuracy of the proposed scheme with Chan's localization algorithm [28] for this realistic SFN scenario. Similar to the pioneering works conducted in [3] and [6], the scatterplot of the location estimates is used to evaluate the positioning accuracy in Fig. 10, whereby each circle

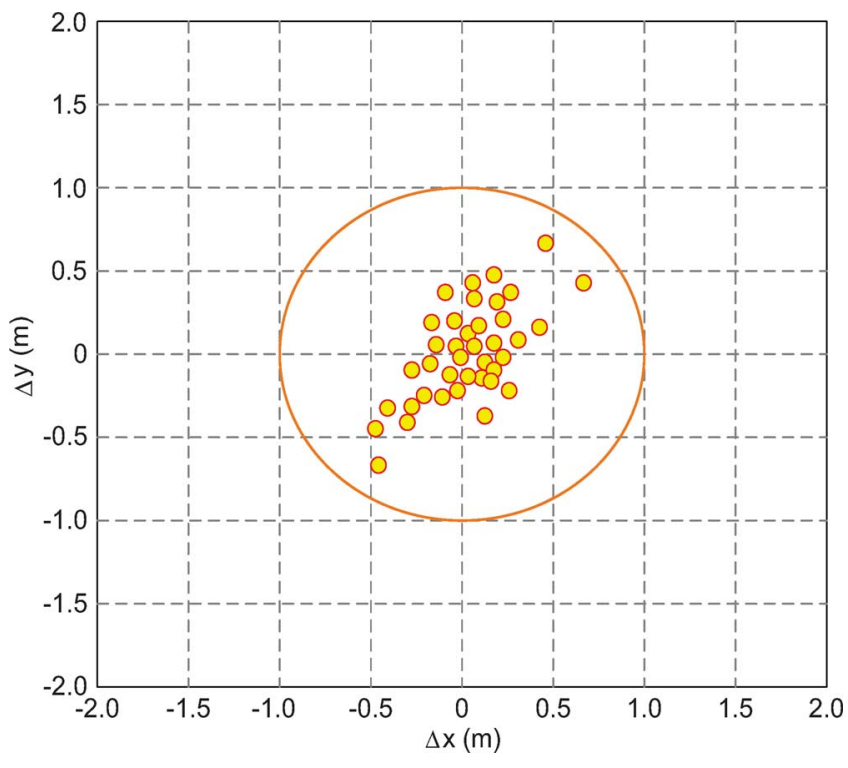

Fig. 10. Positioning accuracy of the proposed scheme in the simulation of the SFN deployed in Hong Kong [32].

denotes one realization of the positioning process, and the positioning accuracy is evaluated by the distance from the estimated location to the user's actual location which is the origin of the coordinates. We observe that the positioning accuracy of less than one meter can be achieved by the proposed scheme. As expected, the excellent ranging accuracy, as demonstrated in Figs. 8 and 9, degraded in this realistic SFN environment and the final positioning accuracy was mainly dependent on the ranging accuracy corresponding to the farthest station with the weakest signal.

\section{CONCLUSIONS}

This paper has proposed a complete positioning solution using the TDS-OFDM signals for the Chinese DTTB standard in the SFN. The TPS embedded in the TDS-OFDM signal is firstly time-division multiplexed to provide orthogonal frequency-domain pilots. The proposed time-frequency positioning scheme jointly utilizes the time-domain PN sequence and the frequency-domain TPS (pilots) for accurate TOA estimation of each transmitter. The theoretical bound of the TOA-based ranging accuracy has been derived. Compared with the-state-of-art methods, the simulation results have shown that the proposed positioning scheme achieves a higher positioning accuracy, while imposing a low computational complexity. Furthermore, backward system compatibility can be maintained. The concept of regarding the TPS as frequency-domain pilots provides a new look at the TDS-OFDM signal structure. In this way, the TPS can be also used to facilitate both the synchronization and channel estimation implemented in the time domain for the TDS-OFDM. Our ongoing work is investigating the challenging problem in which the receiver is located near one particular transmitter and the signals received from all the other transmitters are extremely weak, as well as studying the well-known non-line-of-sight (NLOS) issue in the DTV based positioning systems. 


\section{APPENDIX A}

ProOF OF $E\left\{\nu_{k} \nu_{l}\right\}$ IN (35)

By defining $W_{l}=\Re\left\{W_{l}\right\}+j \Im\left\{W_{l}\right\}=x_{l}+j y_{l}$, where $\Re\{\cdot\}$ denotes the real part and $j=\sqrt{-1}, \nu_{k}$ in (33) can be expressed by (40) on the top of the this page.

$$
\begin{aligned}
\nu_{k}=\Im & \{(\cos ((k+\Delta) \Omega)-j \sin ((k+\Delta) \Omega)) \\
& \times\left(x_{k+\Delta}+j y_{k+\Delta}\right)+(\cos (k \Omega)+j \sin (k \Omega)) \\
& \times\left(x_{k}-j y_{k}\right)+(\cos (\Delta \Omega)-j \sin (\Delta \Omega)) \\
& \left.\times\left(x_{k}-j y_{k}\right)\left(x_{k+\Delta}+j y_{k+\Delta}\right)\right\} \\
= & y_{k+\Delta} \cos ((k+\Delta) \Omega)-x_{k+\Delta} \sin ((k+\Delta) \Omega) \\
& +x_{k} \sin (k \Omega)-y_{k} \cos (k \Omega) \\
+ & \left(x_{k} y_{k+\Delta}-x_{k+\Delta} y_{k}\right) \cos (\Delta \Omega) \\
- & \left(x_{k} x_{k+\Delta}+y_{k} y_{k+\Delta}\right) \sin (\Delta \Omega) .
\end{aligned}
$$

From the definition of the Gaussian white noise $W_{k}$, we have the expectations as shown in (41), where $\delta_{k, l}=1$ if $k=l$, and $\delta_{k, l}=0$ if $k \neq l$.

$$
\begin{aligned}
E\left\{x_{k} \prod_{i=1}^{q} y_{l_{i}}\right\} & =E\left\{y_{k} \prod_{i=1}^{q} x_{l_{i}}\right\}=0, \\
E\left\{x_{k} x_{l}\right\} & =E\left\{y_{k} y_{l}\right\}=\frac{\sigma^{2}}{2} \delta_{k, l}, \\
E\left\{x_{k} x_{l} y_{i} y_{q}\right\} & =\frac{\sigma^{4}}{4} \delta_{k, l} \delta_{i, q}, \\
E\left\{x_{k} x_{l} x_{k+q} x_{l+q}\right\} & =E\left\{y_{k} y_{l} y_{k+q} y_{l+q}\right\}=\frac{\sigma^{4}}{4}\left(\delta_{k, l}\right)^{2} .
\end{aligned}
$$

Therefore, $E\left\{\nu_{k} \nu_{l}\right\}$ can be expressed as in (42). Note that $\delta_{k+\Delta, l+\Delta}=\delta_{k, l}$ has been applied in deriving (42). Obviously, in (42), $\delta_{k, k+\Delta}=\delta_{l, l+\Delta}=\delta_{k, l+\Delta}=\delta_{k+\Delta, l}=0$, since $N / 2<\Delta<N$ and $0 \leq k, l \leq N-1-\Delta$. Thus, we have the final result of $E\left\{\nu_{k} \nu_{l}\right\}$ as shown in (43).

$$
\begin{aligned}
& E\left\{\nu_{k} \nu_{l}\right\}=\frac{\sigma^{2}}{2}\left(\delta_{k, l} \cos ((k+\Delta) \Omega) \cos ((l+\Delta) \Omega)\right. \\
& -\delta_{k+\Delta, l} \cos ((k+\Delta) \Omega) \cos (l \Omega) \\
& +\delta_{k, l} \sin ((k+\Delta) \Omega) \sin ((l+\Delta) \Omega) \\
& -\delta_{k+\Delta, l} \sin ((k+\Delta) \Omega) \sin (l \Omega) \\
& -\delta_{k, l+\Delta} \sin (k \Omega) \sin ((l+\Delta) \Omega) \\
& +\delta_{k, l} \sin (k \Omega) \sin (l \Omega) \\
& -\delta_{k, l+\Delta} \cos (k \Omega) \cos ((l+\Delta) \Omega) \\
& \left.+\delta_{k, l} \cos (k \Omega) \cos (l \Omega)\right)+\frac{\sigma^{4}}{4} \\
& \times\left(\left(\delta_{k, l}\right)^{2}(\cos (\Delta \Omega))^{2}-2 \delta_{k, l+\Delta} \delta_{k+\Delta, l}\right. \\
& \times(\cos (\Delta \Omega))^{2}+\left(\delta_{k, l}\right)^{2}(\cos (\Delta \Omega))^{2} \\
& +\left(\delta_{k, l}\right)^{2}(\sin (\Delta \Omega))^{2}+2 \delta_{k, k+\Delta} \delta_{l, l+\Delta} \\
& \left.\times(\sin (\Delta \Omega))^{2}+\left(\delta_{k, l}\right)^{2}(\sin (\Delta \Omega))^{2}\right) \text {. } \\
& E\left\{\nu_{k} \nu_{l}\right\}=\frac{\sigma^{2}}{2} \delta_{k, l}(\cos ((k+\Delta) \Omega) \cos ((l+\Delta) \Omega) \\
& +\sin ((k+\Delta) \Omega) \sin ((l+\Delta) \Omega)
\end{aligned}
$$

$$
\begin{gathered}
+\sin (k \Omega) \sin (l \Omega)+\cos (k \Omega) \cos (l \Omega)) \\
+\frac{\sigma^{4}}{4} 2\left(\delta_{k, l}\right)^{2}\left((\cos (\Delta \Omega))^{2}+(\sin (\Delta \Omega))^{2}\right) \\
=\sigma^{2} \delta_{k, l} \cos ((k-l) \Omega)+\frac{\sigma^{4}}{2} \delta_{k, l} .
\end{gathered}
$$

\section{REFERENCES}

[1] E. D. Kaplan and C. Hegarty, Understanding GPS: Principles and Applications, Second ed. Boston, MA: Artech House, 2005.

[2] B. T. Sieskul, F. Zheng, and T. Kaiser, "A hybrid SS-ToA wireless NLoS geolocation based on path attenuation: ToA estimation and CRB for mobile position estimation," IEEE Trans. Veh. Technol., vol. 458, no. 9, pp. 4930-4942, Nov. 2009.

[3] M. Rabinowitz and J. Spilker, "A new positioning system using television synchronization signals," IEEE Trans. Broadcast., vol. 51, no. 1, pp. 51-61, Mar. 2005.

[4] P. Kovar and F. Vejrazka, "Multi system navigation receiver," in Proc. IEEE/ION Position, Loc. Nav. Symp. (PLANS), Monterey, CA, May 5-8, 2008, pp. 860-864.

[5] X. Wang, Y. Wu, and B. Caron, "Transmitter identification using embedded pseudo random sequences," IEEE Trans. Broadcast., vol. 50 , no. 3, pp. 244-252, Sep. 2004.

[6] X. Wang, Y. Wu, and J. Chouinard, "A new position location system using DTV transmitter identification watermark signals," EURASIP Journal on Applied Signal Processing, vol. 2006, pp. 1-11, 2006.

[7] D. Serant, P. Thevenon, M. L. Boucheret, O. Julien, C. Macabiau, S. Corazza, M. Dervin, and L. Ries, "Development and validation of an OFDM/DVB-T sensor for positioning," in Proc. IEEE/ION Position Location and Navigation Symposium (PLANS'10), Indian Wells, USA, May 4-6, 2010, pp. 988-1001.

[8] Framing Structure, Channel Coding and Modulation for Digital Television Terrestrial Broadcasting System., Chinese National Standard, GB 20600-2006, Aug. 2006.

[9] O. Bar-Shalom and A. J. Weiss, "Efficient direct position determination of orthogonal frequency division multiplexing signals," IET Radar Sonar and Navigation, vol. 3, no. 2, pp. 101-111, Jun. 2009.

[10] C. Mensing, S. Plass, and A. Dammann, "Synchronization algorithms for positioning with OFDM communications signals," in Proc. 4th Workshop on Positioning, Nav. Commun., Hannover, Mar. 22, 2007, pp. 205-210.

[11] R. K. Martin, J. S. Velotta, and J. F. Raquet, "Bandwidth efficient cooperative TDOA computation for multicarrier signals of opportunity," IEEE Trans. Signal Process., vol. 57, no. 6, pp. 2311-2322, Jun. 2009.

[12] R. K. Martin, C. Yan, and H. H. Fan, "Bounds on distributed TDOAbased localization of OFDM sources," in Proc. ICASSP, Taipei, China, Apr. 19-24, 2009, pp. 2289-2292.

[13] X. Li and K. Pahlavan, "Super-resolution TOA estimation with diversity for indoor geolocation," IEEE Trans. Wireless Commun., vol. 3 , no. 1, pp. 224-234, Jan. 2004.

[14] T. J. S. Khanzada, A. R. Ali, and A. S. Omar, "Time difference of arrival estimation using super resolution algorithms to minimize distance measurement error for indoor positioning systems," in Proc. IEEE Int. Conf. Multitopic Conf. (INMIC), Karachi, Pakistan, Dec. 23/24, 2008, pp. $443-447$

[15] T. Becker, F. Kuchen, and W. Wiesbeck, "A new network planning approach for digital audio broadcasting," IEEE Trans. Veh. Technol., vol. 48, no. 2, pp. 619-626, Mar. 1999.

[16] Z. Yang, L. Dai, J. Wang, and Z. Wang, "Transmit diversity for TDSOFDM broadcasting system over doubly selective fading channels," IEEE Trans. Broadcast., vol. 57, no. 1, pp. 135-142, Mar. 2011.

[17] C. yen Ong, J. Song, C. Pan, and Y. Li, "Technology and standards of digital television terrestrial multimedia broadcasting," IEEE Commun. Mag., vol. 48, no. 5, pp. 119-127, May 2010.

[18] J. Wang, Z. Yang, C. Pan, and J. Song, "Iterative padding subtraction of the PN sequence for the TDS-OFDM over broadcast channels," IEEE Trans. Consum. Electron., vol. 51, no. 11, pp. 1148-1152, Nov. 2005.

[19] L. Dai, Z. Wang, and S. Chen, "A novel uplink multiple access scheme based on TDS-FDMA," IEEE Trans. Wireless Commun., vol. 10, no. 3, pp. 757-761, Mar. 2011.

[20] C. Wang, M. Patzold, and Q. Yao, "Stochastic modeling and simulation of frequency-correlated wideband fading channels," IEEE Trans. Veh. Technol., vol. 56, no. 3, pp. 1050-1063, May 2007. 
[21] J. Song, Z. Yang, L. Yang, K. Gong, C. Pan, J. Wang, and Y. Wu, "Technique review on Chinese digital terrestrial television broadcasting standard and measurements on some working modes," IEEE Trans. Broadcast., vol. 53, no. 1, pp. 1-7, Mar. 2007.

[22] L. Dai, Z. Wang, J. Wang, and Z. Yang, "Joint channel estimation and time-frequency synchronization for uplink TDS-OFDMA systems," IEEE Trans. Consum. Electron., vol. 56, no. 2, pp. 494-500, May 2010.

[23] U. Ladebusch and C. A. Liss, "Terrestrial DVB (DVB-T): A broadcast technology for stationary portable and mobile use," Proc. IEEE, vol. 94, no. 1, pp. 183-193, Jan. 2006.

[24] D. K. Kim, S. H. Do, H. B. Cho, H. J. Chol, and K. B. Kim, “A new joint algorithm of symbol timing recovery and sampling clock adjustment for OFDM systems," IEEE Trans. Consum. Electron., vol. 44, no. 3, pp. 1142-1149, Aug. 1998.

[25] M. Speth, S. Fechtel, G. Fock, and H. Meyr, "Optimum receiver design for OFDM-based broadband transmission-part II: A case study," IEEE Trans. Commun., vol. 49, no. 4, pp. 571-578, Apr. 2001.

[26] A. Sayed, A. Tarighat, and N. Khajehnouri, "Network-based wireless location: Challenges faced in developing techniques for accurate wireless location information," IEEE Signal Process. Mag., vol. 22, no. 4, pp. 24-40, Jul. 2005.

[27] B. T. Fang, "Simple solutions for hyperbolic and related position fixes," IEEE Trans. Aerosp. Electron. Syst., vol. 26, no. 5, pp. 748-753, Sep. 1990.

[28] Y. T. Chan and K. C. Ho, "A simple and efficient estimator for hyperbolic location," IEEE Trans. Signal Process., vol. 42, no. 8, pp. 1905-1915, Aug. 1994.

[29] W. H. Foy, "Position-location solutions by Taylor-series estimation," IEEE Trans. Aerosp. Electron. Syst., vol. 12, no. 2, pp. 187-194, Mar. 1976.

[30] P. Tichavsky and P. Handel, "Efficient tracking of multiple sinusoids with slowly varying parameters," in Proc. ICASSP, Minneapolis, MN, Apr. 27-30, 1993, pp. 368-371.

[31] Guideline for Evaluation of Radio Transmission Technology for IMT2000., Recommendation ITU-R M.1225, 1997.

[32] Deployment of DTMB in Single Frequency Network of Hong Kong., ITU-R 31/6, Document 6A/499-E, Apr. 2011.

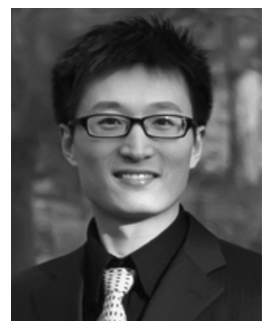

Linglong Dai received the Ph.D. degree from the Department of Electronic Engineering, Tsinghua University, Beijing, China, in 2011.

He is now a Post Doctoral Fellow with the Department of Electronic Engineering as well as the Tsinghua National Laboratory of Information Science and Technology (TNList), Tsinghua University. He has been actively involved in the development of Chinese DTTB evolution standard. His research interests lie in the field of synchronization, channel estimation for wireless communication system, space-time coding and diversity techniques, multiple access techniques, and wireless positioning as well.

Dr. Dai was awarded the Outstanding Doctor of Electronic Engineering and the Academic Star of Tsinghua University.

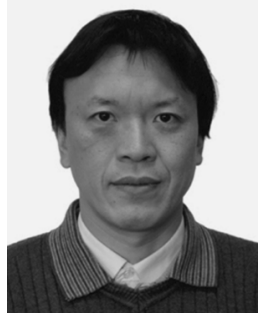

Zhaocheng Wang (SM'10) received the B.S., M.S. and $\mathrm{Ph} . \mathrm{D}$. degrees from Tsinghua University, Beijing, China, in 1991, 1993, and 1996, respectively.

From 1996 to 1997, he was with Nanyang Technological University (NTU), Singapore as a Post Doctoral Fellow. From 1997 to 1999, he was with OKI Techno Centre, Singapore, Pte. Ltd., firstly as a Research Engineer and then as a Senior Engineer. From 1999 to 2009, he worked at SONY Deutschland $\mathrm{GmbH}$, first as a Senior Engineer and then as a Principal Engineer. He is currently a Professor at the Department of Electronic Engineering, Tsinghua University. His research areas include wireless communications, digital broadcasting and millimeter wave communications. He holds 22 granted U.S./EU patents and has published over 60 technical papers. He has served as technical program committee co-chair/member of many international conferences. He is a Senior Member of IEEE and a Fellow of IET.

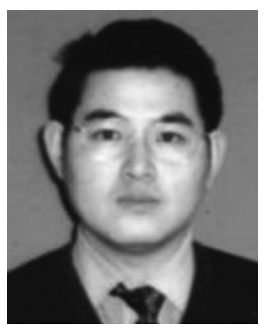

Changyong Pan (M’01) is an Associate Professor in the Department of Electronic Engineering and Deputy Director of the DTV R\&D Center of Tsinghua University, Beijing. China. He works as a member of a Chinese ITU-R delegation group for the Chinese DTTB standard, representing China to present at Geneva in the DTV area several times and is now the contact person for DTTB-related issues. He is a key working group member for the serial national equipment standards related to the DTTB. He has authored or coauthored more than 140 technical papers and published two technical books. He holds 24 patents. $\mathrm{He}$ is actively involved in the Chinese DTTB standard process as one of major technical contributors, including algorithm development, system design, hardware implementation, and standard drafting, especially all field trials and related system-level $R \& D$

Mr. Pan has won the 2nd national technical invention award two times, and is also the winner of numerous other awards.

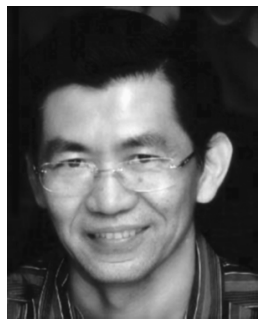

Sheng Chen (M'90-SM'97-F'08) received the B.Eng. degree from Huadong Petroleum Institute, Dongying, China, in 1982, and the Ph.D. degree from the City University, London, U.K., in 1986, both in control engineering, and was awarded the Doctor of Sciences (D.Sc.) degree by the University of Southampton, Southampton, U.K., in 2004.

From October 1986 to August 1999, he held research and academic appointments at the University of Sheffield, Sheffield, U.K., the University of Edinburgh, Edinburgh, U.K., and the University of Portsmouth, Portsmouth, U.K. Since September 1999, he has been with the Electronics and Computer Science, the University of Southampton, where he currently holds the post of Professor of Intelligent Systems and Signal Processing. He is a Distinguished Adjunct Professor at King Abdulaziz University, Jeddah, Saudi Arabia. His research interests include wireless communications, adaptive signal processing for communications, machine learning, evolutionary computation methods, and intelligent control systems. He has published over 450 research papers.

Dr. Chen is a Chartered Engineer (C.Eng.) and a Fellow of IET. In the database of the world's most highly cited researchers, compiled by Institute for Scientific Information (ISI) of the USA, Dr. Chen is on the list of the highly cited researchers in the engineering category (March 2004). 